\title{
Erratum to: Nanocomposite quasi-solid-state electrolyte for highsafety lithium batteries
}

Hyunji Choi ${ }^{1, \S}$, Hyun Woo Kim ${ }^{1, \S}$, Jae-Kwang $\operatorname{Kim}^{2}(\varangle)$, Young Jun $\operatorname{Lim}^{1}$, Youngsik Kim ${ }^{1}(\varangle)$, and Jou-Hyeon $\mathrm{Ahn}^{3}(\bowtie)$

${ }^{1}$ School of Energy \& Chemical Engineering, Ulsan National Institute of Science and Technology (UNIST), Ulsan 689-798, Republic of Korea

${ }^{2}$ Department of Solar \& Energy Engineering, Cheongju University, Cheongju, Chungbuk 28503, Republic of Korea

${ }^{3}$ Department of Materials Engineering and Convergence Technology and Research Institute for Green Energy Convergence Technology, Gyeongsang National University, 900, Gajwa-dong, Jinju 660-701, Republic of Korea

${ }^{\S}$ Hyunji Choi and Hyun Woo Kim contributed equally to this work.

(C) Tsinghua University Press and Springer-Verlag Berlin Heidelberg 2017

\section{Erratum to}

Nano Research 2017, 10(9): 3092-3102

DOI 10.1007/s12274-017-1526-2

The third affiliation of the authors in the original version of this article was unfortunately wrongly written on page 3092 and the first page of the ESM.

Instead of

${ }^{3}$ School of Energy \& Chemical Engineering and Research Institute for Green Energy Convergence Technology, Gyeongsang National University, 900, Gajwa-dong, Jinju 660-701, Republic of Korea

It should read

${ }^{3}$ Department of Materials Engineering and Convergence Technology and Research Institute for Green Energy Convergence Technology, Gyeongsang National University, 900, Gajwa-dong, Jinju 660-701, Republic of Korea 